\title{
Wuchang Fangcang Shelter Hospital: Practices, Experiences, and Lessons Learned in Controlling COVID-19
}

\author{
Bingzheng Shen ${ }^{1,2} \cdot$ Li Chen $^{1,2} \cdot$ Lu Zhang $^{1,2} \cdot$ Mengke Zhang ${ }^{1,2} \cdot$ Jing $\mathrm{Li}^{1,2} \cdot$ Jie $\mathrm{Wu}^{1,2} \cdot \mathrm{Kunlin}^{\mathrm{Chen}}{ }^{1,2} \cdot$ \\ Yuanguo Xiong ${ }^{1,2} \cdot$ Wei Song $^{1} \cdot$ Benhong Zhou ${ }^{1}$
}

Accepted: 23 June 2020 / Published online: 4 July 2020

(C) Springer Nature Switzerland AG 2020

\begin{abstract}
In early January 2020, the outbreak of the new corona virus pneumonia (Corona Virus Disease 2019, COVID-19) occurred. Wuhan, the capital city of Hubei province, became the epicenter of the disease in China. The rapid growth of patients had exceeded the maximum affordability of local medical resources. A large comprehensive gymnasium was converted into Wuchang Fangcang Shelter Hospital in order to provide adequate medical beds and appropriate care for the confirmed patients with mild to moderate symptoms. For these hospitalized patients with COVID-19, medication became the mainstay of therapy. From 5th February to 10th March, a team of pharmacists successfully completed drug supplies and pharmaceutical services for 1124 patients and approximately 800 medical staff, and, while doing so, received zero complaint, and experienced zero disputes and zero pharmacist infection. This paper summarizes the development and construction of the pharmacy, human resource allocation of pharmacists, pharmacy administration, and pharmaceutical services. It aims to review a 34-day period of pharmaceutical practice and serve as a reference for other health professionals working on COVID-19 prevention and treatment in other regions.
\end{abstract}

Keywords Wuchang Fangcang Shelter Hospital · Pharmacy administration · Pharmaceutical service · Occupational protection · COVID-19

On 8th December 2019, a confirmed case of the new coronavirus infection of pneumonia, termed Corona Virus Disease 2019 (COVID-19), was detected in Wuhan City [1]. In a short period of time, the virus spread quickly throughout the country, and the number of infected patients increased rapidly. At the beginning of February 2020, available hospital beds soon reached full occupancy in those hospitals designated for antivirus treatment. To complicate matters further, some medical workers were infected due to occupational exposure, which

This article is part of the Topical Collection on Covid-19

Electronic supplementary material The online version of this article (https://doi.org/10.1007/s42399-020-00382-1) contains supplementary material, which is available to authorized users.

Benhong Zhou

benhongzh@163.com

1 Department of Pharmacy, Renmin Hospital of Wuhan University, No.238 Jiefang Road, Wuchang, Wuhan, China

2 Department of Pharmacy, Wuchang Fangcang Shelter Hospital, Hongshan Gymnasium, No.1 Tiyuguan Road, Wuchang, Wuhan, China forced the medical team into quarantine for medical observation. Based on clinical manifestations, confirmed patients are divided into mild, moderate, severe, and critical types $[2,3]$. Since more than $80 \%$ of COVID-19 patients were mild or moderate types [4-6], a novel public health measure, Fangcang Shelter Hospitals, was conceived [7]. In case of emergency, these temporary hospitals have been able to provide extra beds capacity at short notice and provide classified treatments. All confirmed patients with mild and moderate symptoms could be admitted to the Fangcang Shelter Hospital for free medical treatment. During the worst epidemic period in Wuhan, a total of 16 Fangcang Shelter Hospital were established. Wuchang Fangcang Shelter Hospital was developed from the Hongshan Gymnasium and was one of the first three hospitals accepting patients and was the last one to be closed. It covered an area of $14,800 \mathrm{~m}^{2}$ and housed a total of 800 beds, which were separated across three independent regions in order to optimize management and treatment efficiency. During this major public health emergency, pharmacists, as a member of the medical team, have been responsible for providing professional and superior pharmaceutical services. This paper looks back at the pharmacy construction, 
occupational protection, pharmacy administration, and pharmaceutical services at Wuchang Fangcang Shelter Hospital. These practices and lessons at the forefront of containing the virus may help others in their efforts around the world.

\section{Location, Arrangement, and Allocation of Human Resources}

The pharmacy of Wuchang Fangcang Shelter Hospital was a conversion from two referee meeting rooms in Hongshan Gymnasium (Fig. $1 \mathrm{a}$ and b). The two rooms were adjacent and connected by a shuttle door. One room was used as pharmacy, the other served as a level 2 warehouse (Fig. $1 \mathrm{c}$ and d). Due to space constraints, there was no room for a level 2 warehouse for medications requiring refrigeration. All central air conditioners were turned off to prevent the virus spreading through the ventilation systems. Several household heaters, humidifiers, air purifiers, and refrigerators were used to maintain the appropriate temperature, humidity, and clean air for the storage of medicines.

Ten pharmacists from Renmin Hospital attached to Wuhan University provided strong support and formed a professional pharmaceutical team (Table 1). The pharmacy was open 24/7 (pharmacists working 12-h shifts), enabling the constant availability of pharmaceutical services at all times. Considering the long hours, high-intensity, and complexity, most pharmacists working in Wucang Fangcang Shelter Hospital were young to middle-aged, well-educated with a master's degree or higher, and with at least 5 years of pharmacy experience.

\section{Occupational Protection}

The team of pharmacists was monitored regularly. Body temperature was measured twice a day, and nucleic acid and specific antibodies of COVID-19 were tested twice per month. Attention was also paid to the staff's mental and emotional health. If necessary, psychological evaluations were carried out, and psychological counseling was provided. The correct use of personal protective equipment (PPE) and regular and thorough hand hygiene were key measures in the prevention and control against infection through contact transmission, droplet transmission, and airborne virus particles. According to the risk of exposure in different working areas, pharmacists took different precautions (Table 2) [8]. Various protective measures were taken to ensure that pharmacists provided professional services in a responsible manner.

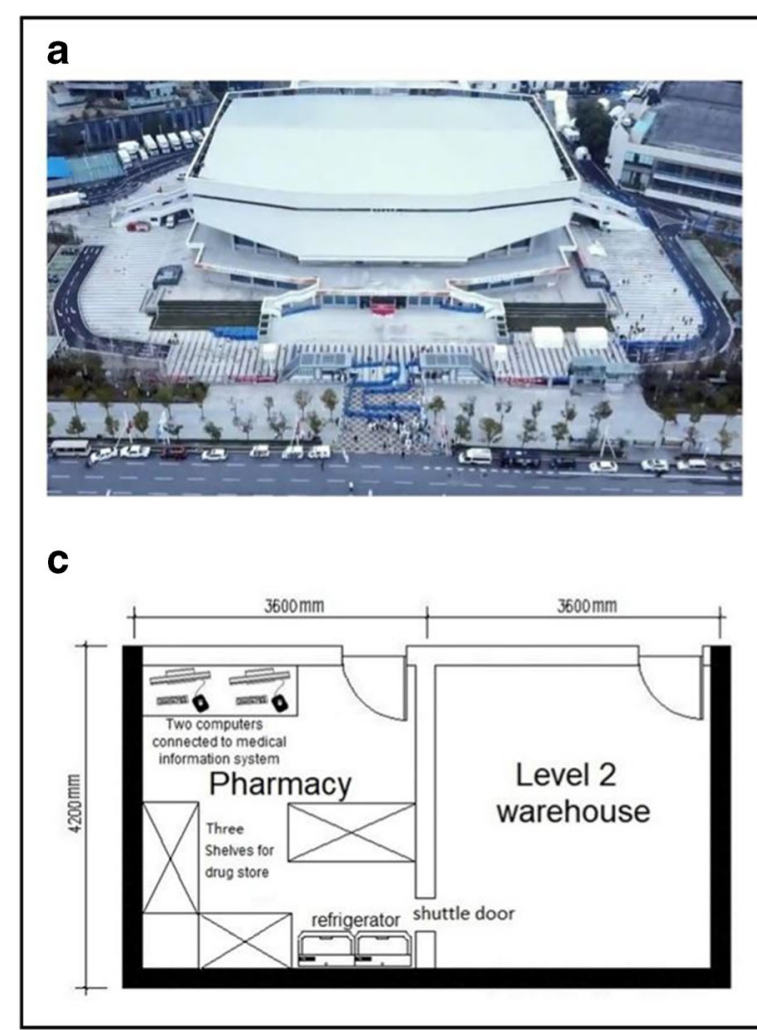

Fig. 1 Wucang Fangcang Shelter Hospital and its pharmacy. a The overall view of Wucang Fangcang Shelter Hospital rebuilt from Hongshan Gymnasium. b One of three independent regions for

\section{b}

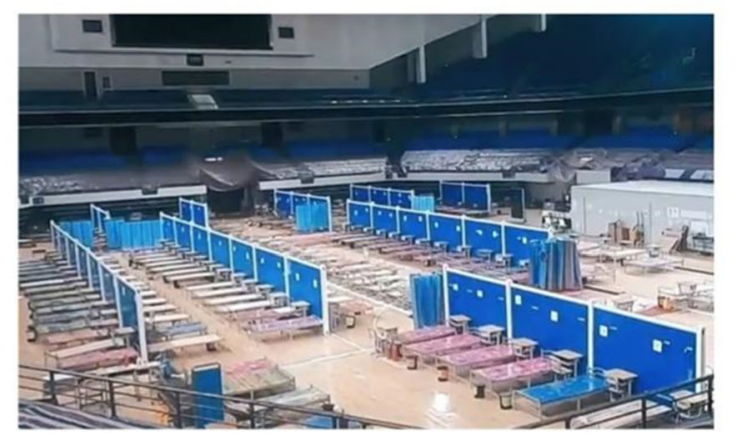

d

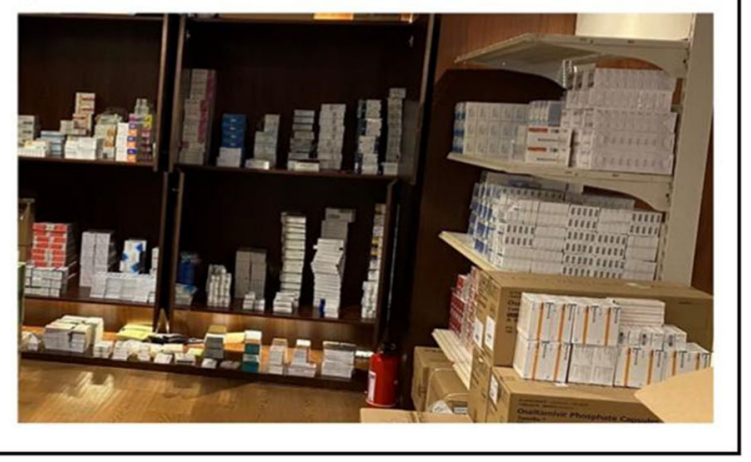

confirmed patients with mild or moderate symptom. c Layout of pharmacy reconstructed from meeting room. d Medicines on pharmacy shelves for patients, medical staffs and logistics support workers 
Table 1 Human resources structure of pharmacists in Wucang Fangcang Shelter Hospital

\begin{tabular}{lll}
\hline Pharmacist characteristics & Number & Rate $(\%)$ \\
\hline Gender & 7 & 70 \\
Male & 3 & 30 \\
Female & & \\
Age & 1 & 10 \\
$50 \sim 60$ & 1 & 10 \\
$40 \sim 50$ & 8 & 80 \\
$30 \sim 40$ & & \\
Degree & 6 & 60 \\
Doctor & 4 & 40 \\
Master & 0 & 0 \\
Bachelor & & \\
Professional title & 1 & 10 \\
Chief pharmacist/professor & 1 & 70 \\
Associate chief pharmacist & 7 & 10 \\
Pharmacist-in-charge & 1 & \\
Pharmacist & &
\end{tabular}

\section{Drug Supply and Pharmacy Administration}

All medications used in Wucang Fangcang Shelter Hospital were obtained from three sources:

1. Renmin Hospital of Wuhan University, which acted as the National general coordinating agency for all medical teams, provided medicines for chronic diseases and firstaid.

2. Some medicines recommended in the diagnosis and treatment guidelines were obtained from Wuhan epidemic prevention and control headquarters [9].

3. Medicines donated by pharmaceutical companies or charitable organization.

The diverse sources of medications added to the need for efficient and accurate pharmacy work, caused many difficulties for the pharmacy administration. The corresponding work processes, including the receipt of medicines, selection, inspection, prescription-checking dispensing, and distribution were established under the unified leadership of Wuchang Fangcang Shelter Hospital (Fig. 2).

According to their characteristics and according to the diagnosis and treatment guidelines, other regulatory documents $[9,10]$, combined with expert clinical opinions, a list of medicines was determined for infected patients with mild and moderate symptoms. This involved symptomatic treatment, prevention of complications, treatment of underlying diseases, emergency rescue medications, and so on. Based on the actual clinical situation, the variety and quantity of medication were regularly adjusted and supplemented. A catalog containing 116 medications was finally revised in 4 Mar 2020 and showed in Table S1.

The category of medicines in the pharmacy was compatible with the function of the Fangcang Shelter Hospital, and covered antiviral, antibacterial, antipyretic, antitussive,

Table 2 Protection measures for pharmacists in Wucang Fangcang Shelter Hospital

\begin{tabular}{|c|c|c|}
\hline Risk classification & Pharmacist activities & Protective measures \\
\hline $\begin{array}{l}\text { High risk } \\
\text { - Contaminated area }\end{array}$ & $\begin{array}{l}\text { Contact with confirmed patients: } \\
\text { Medication distribution } \\
\text { Medication counseling } \\
\text { Pharmaceutical care } \\
\text { Contact with clinical specimens: } \\
\text { Detection of therapeutic drug concentration } \\
\text { Gene typing detection for drug metabolism }\end{array}$ & $\begin{array}{l}\text { Hand hygiene } \\
\text { Protective clothing } \\
\text { Isolation clothing } \\
\text { Protective mask-N95 } \\
\text { Work cap } \\
\text { Goggle and face shield } \\
\text { Double gloves } \\
\text { Boot covers } \\
\text { Shoe covers }\end{array}$ \\
\hline $\begin{array}{l}\text { Medium risk } \\
\text { - Potential contamination area }\end{array}$ & $\begin{array}{l}\text { Contact with medical staffs working in the ward } \\
\text { Discussing medication options with doctor } \\
\text { Explaining medication precautions to doctor/nurse } \\
\text { Distributing medication to staffs with illness (non-COVID-19) } \\
\text { Contact with medical staffs working outside the ward } \\
\text { Distributing medication to nurse } \\
\text { Replacing damaged medication (outside contaminated area) }\end{array}$ & $\begin{array}{l}\text { Hand hygiene } \\
\text { Work clothing } \\
\text { Isolation clothing } \\
\text { Surgical or N95 mask } \\
\text { Work cap } \\
\text { Goggle and face shield } \\
\text { Gloves }\end{array}$ \\
\hline $\begin{array}{l}\text { Low risk } \\
\text { - Clean area }\end{array}$ & $\begin{array}{l}\text { Non-contact work } \\
\text { Checking prescription based on } 5 \mathrm{G} \text { remote network } \\
\text { Releasing drug information through social software } \\
\text { Pharmaceutical education for patients through broadcast } \\
\text { Getting medicines from level } 1 \text { warehouse }\end{array}$ & $\begin{array}{l}\text { Hand hygiene } \\
\text { Work clothing } \\
\text { Surgical mask } \\
\text { Work cap }\end{array}$ \\
\hline
\end{tabular}


antianxietic, expectorant, and chronic disease medications. It conformed the treatment protocols for COVID-19, and met the needs of some patients with underlying disease, as well as including first-aid medicines to be able to deal with any sudden incidents.

To integrate traditional Chinese and Western medicine to treat infected patients, 16 kinds of Traditional Chinese Medication, including preventive and therapeutic decoctions for medical staffs and patients (Fig. S1), respectively, were also available.

\section{Pharmaceutical Services}

In addition to maintaining a constant and regular supply of drugs, the provision of pharmaceutical services was another important and indispensable duty during the pandemic [11-13]. Relying on the 5G network and medical information systems, the team of pharmacists accomplished pharmaceutical services smoothly, helping to reduce the risk of occupational exposure in the Shelter Hospital.
Pharmaceutical services focused on the following six aspects:

1. Checking medical orders related to the drug therapy

2. Paying attention to the prescriptions for patients with underlying diseases

3. The suitability of the usage and dosage of the medication

4. Monitoring adverse drug reactions and drug-drug interactions

5. Summarizing and sharing the latest drug information

6. Providing medication-related consultation and education.

In some cases, several patients with chronic diseases took repeated medications or overdose. They were admitted to the hospital with their own chronic disease medicines. Not clearly understanding the situation, doctors prescribed the same medication or other drugs possessing the same pharmacological effect. To overcome this problem, patients taking chronic disease medicines (such as antihypertensive drugs and hypoglycemic agents) were screened out by the pharmacy information system. As a result, pharmacists could intervene in time on medical orders and prescriptions. The pharmacists also

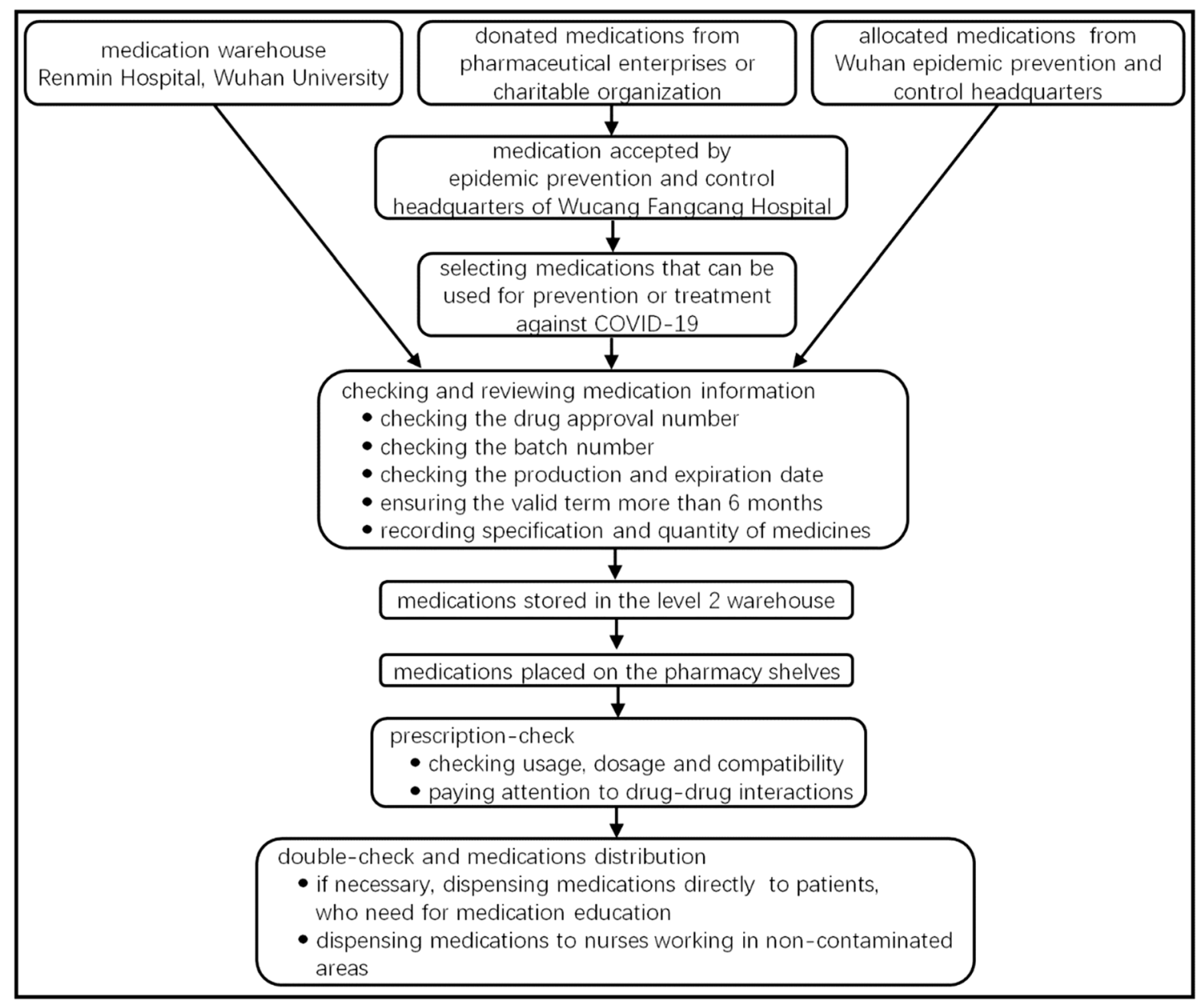

Fig. 2 Workflow for pharmacists in Wuchang Fangcang Shelter Hospital 
provided their service to medical staff and logistics support workers, dispensing medicines as prescribed by doctors on duty or by authorized medical team leaders.

\section{Conclusion}

While our understanding of the virus deepens and with the constant improvement of diagnosis and treatment and strategies for prevention and control, pharmacists should continue to actively collect and improve their services based on the latest information. For instance, it has recently been discussed whether or not angiotensin-converting enzyme inhibitor (ACE-i) and angiotensin receptor blockers (ARBs) increased the susceptibility of COVID-19. In the pharmaceutical services provided at Wuchang Fangcang Shelter Hospital, ACE-I and ARBs were not recommended, but now, the latest joint viewpoint from three U.S. heart groups states that patients with COVID-19 should take ACE inhibitors and ARBs [14].

Not to be neglected, the mental health of the pharmacist also requires close attention. Having worked in this shelter hospital for 23 days, one pharmacist felt anxious and uncomfortable. Tests of viral-specific nucleic acid and antibody were negative and computed tomography reported normal results. After psychological counseling and a brief period of rest, the physical and mental states were greatly improved, and the pharmacist gradually recovered. Psychology-related research shows that during the peak of the COVID-19 epidemic in China, more than one-third of medical staffs suffer from insomnia, which may progress to depression, anxiety, and stress trauma [15].

On March 10, 2020, Wucang Fangcang Shelter Hospital was closed, thus indicating that all of these large-scale temporary hospitals had completed their missions in Wuhan. The team of pharmacists has done their utmost efforts to perform their professional duties, ensuring the supply of medicines and providing high-level pharmaceutical services. Currently, the COVID-19 outbreak is spreading worldwide, and the situation awaits a vaccine. Pharmacists should unite globally to contribute their expertise and strength to help prevent the spread of the virus.

Acknowledgments In this concerted effort against the COVID-19 virus, thousands of medical staffs in China (doctors, pharmacists, nurses, inspection, and image technicians) put their hearts and souls into curing infected patients, subjecting themselves to huge risk of infection. We thank all pharmacists for their valuable suggestions and great contributions in fighting this epidemic disease. Thanks also to all logistics support workers in delivering invaluable protection equipment and living supplies to medical staffs and patients.

Authors' Contributions BS and BZ initiated the topic. BS, BZ, LC, LZ, MZ, JL, JW, KC, YX, and WS participated in discussions. BS wrote the first draft of the manuscript. All authors read and approved the final manuscript.

\section{Compliance with Ethical Standards}

Conflict of Interest The authors declared that they have no conflict of interest.

Ethics Statements Not applicable. This is a descriptive and retrospective study that and does not undermine the principles according to the ethical standards of the institutional and/or national research committee and/or the 1964 Helsinki declaration and its later amendments or comparable ethical standards.

\section{References}

1. Guan WJ, Ni ZY, Hu Y, Liang WH, Ou CQ, He JX, et al. Clinical characteristics of Coronavirus disease 2019 in China. N Engl J Med. 2020;382(18):1708-20.

2. Chan JF, Yuan S, Kok KH, To KK, Chu H, Yang J, et al. A familial cluster of pneumonia associated with the 2019 novel coronavirus indicating person-to-person transmission: a study of a family cluster. Lancet. 2020;395(10223):514-23.

3. Office of State Administration of Traditional Chinese Medicine. Diagnosis and treatment protocol for COVID-19 (trial version 7). 2020. Available at: http://ghs.satcm.gov.cn/gongzuodongtai/202003-20/14089.html. Accessed May 202020.

4. Zu ZY, Jiang MD, Xu PP, Chen W, Ni QQ, Lu GM, et al. Coronavirus disease 2019 (COVID-19): a perspective from China. Radiology. 2020;200490.

5. Li H, Liu SM, Yu XH, Tang SL, Tang CK. Coronavirus disease 2019 (COVID-19): current status and future perspectives. Int J Antimicrob Agents. 2020;55(5):105951.

6. Yang W, Cao Q, Qin L, Wang X, Cheng Z, Pan A, et al. Clinical characteristics and imaging manifestations of the 2019 novel coronavirus disease (COVID-19): a multi-center study in Wenzhou city, Zhejiang, China. J Infect. 2020;80(4):388-93.

7. Chen S, Zhang Z, Yang J, Wang J, Zhai X, Bärnighausen T, et al. Fangcang shelter hospitals: a novel concept for responding to public health emergencies. Lancet. 2020;395(10232):1305-14.

8. Chinese Pharmaceutical Association. Coronavirus SARS-CoV-2 infection: expert consensus of guidance, prevention and control strategy for hospital pharmacy work (the Second Edition). 2020. Available at: http://www.cpa.org.cn/cpadmn/attached/file/ 20200216/1581852960473177.pdf. Accessed 1 April 2020.

9. China National Health Commission. Diagnosis and treatment guidelines for the new Coronavirus infected pneumonia (theSeventh Edition). 2020. Available at: http://www.nhc.gov.cn/ yzygj/s7653p/202003/46c9294a7dfe4cef80dc7f5912eb1989. shtml. Accessed 20 May 2020.

10. International Pharmaceutical Federation. Coronavirus 2019-nCoV outbreak: information and interim guidelines for pharmacists and the pharmacy workforce. 2020. Available at: http://www.cpa.org. $\mathrm{cn} / \mathrm{cpadmn} /$ attached/file/20200207/1581008981375538.pdf. Accessed 20 May 2020.

11. Liu S, Luo P, Tang M, Hu Q, Polidoro JP, Sun S, et al. Providing pharmacy services during the coronavirus pandemic. Int J Clin Pharm. 2020;42(2):299-304.

12. Meng L, Qiu F, Sun S. Providing pharmacy services at cabin hospitals at the coronavirus epicenter in China. Int J Clin Pharm. 2020;42(2):305-8.

13. Li H, Zheng S, Liu F, Liu W, Zhao R. Fighting against COVID-19: innovative strategies for clinical pharmacists. Res Social Adm Pharm. 2020;S1551-7411(20):30328-4.

14. Patel AB, Verma A. COVID-19 and angiotensin-converting enzyme inhibitors and angiotensin receptor blockers: what is 
the evidence? JAMA. 2020. Available at: https://jamanetwork. com/journals/jama/fullarticle/2763803. Accessed 20 May 2020.

15. Zhang C, Yang L, Liu S, Ma S, Wang Y, Cai Z, et al. Survey of insomnia and related social psychological factors among medical staff involved in the 2019 novel coronavirus disease outbreak. Front Psychiatry. 2020;11:306.

Publisher's Note Springer Nature remains neutral with regard to jurisdictional claims in published maps and institutional affiliations. 